\title{
Dielectrophoretic characterization of dendritic cell deformability upon maturation
}

\author{
Anoop Menachery $\star, \ddagger, 1$ iD, Jiranuwat Sapudom ${ }^{\ddagger}, 2$ iD, Abhishek Vembadi ${ }^{3}$, Aseel Alatoom², Jeremy Teo**,2,4 iD \& Mohammad A \\ Qasaimeh***,3,4 iD \\ ${ }^{1}$ Institute of Engineering \& Transport, Electrical \& Electronics, Malta College for Arts, Science \& Technology, Malta; ${ }^{2}$ Laboratory for Immuno Bioengineering Research \\ \& Applications, Division of Engineering, New York University Abu Dhabi, Abu Dhabi, UAE; ${ }^{3}$ Advanced Microfluidics \& Microdevices Laboratory, Division of Engineering, \\ New York University Abu Dhabi, Abu Dhabi, UAE; ${ }^{4}$ Department of Mechanical Engineering, Tandon School of Engineering, New York University, NY, USA; *Author for \\ correspondence: anoop.menachery@mcast.edu.mt; **Author for correspondence: jeremy.teo@nyu.edu; ${ }^{\star \star \star A u t h o r ~ f o r ~ c o r r e s p o n d e n c e: m o h a m m a d . q a s a i m e h @ n y u . e d u ; ~}$ \\ ${ }_{\ddagger}^{\ddagger}$ Authors contributed equally
}

BioTechniques 70: 29-36 (January 2021) 10.2144/btn-2020-0126

First draft submitted: 19 August 2020; Accepted for publication: 15 October 2020; Published online: 3 November 2020

\section{ABSTRACT}

We have developed a rapid technique for characterizing the biomechanical properties of dendritic cells using dielectrophoretic forces. It is widely recognized that maturing of dendritic cells modulates their stiffness and migration capabilities, which results in T-cell activation triggering the adaptive immune response. Therefore it is important to develop techniques for mechanophenotyping of immature and mature dendritic cells. The technique reported here utilizes nonuniform electric fields to exert a substantial force on the cells to induce cellular elongation for optical measurements. In addition, a large array of interdigitated electrodes allows multiple cells to be stretched simultaneously. Our results indicate a direct correlation between F-actin activity and deformability observed in dendritic cells, determined through mean fluorescence signal intensity of phalloidin.

\section{METHOD SUMMARY}

A device with an array of interdigitated microelectrodes was developed using standard photolithography approaches. The dendritic cells used were differentiated human leukemic cells. Differentiated dendritic cells were treated with cytochalasin B to decrease F-actin expression. Subsequently, cells were detached and used for dielectrophoresis experimentation and F-actin quantification. Suspended cells were loaded to the device, then immobilized and stretched using a multistep dielectrophoresis approach. Cell deformation was measured from microscopic images.

\section{KEYWORDS:}

actin $\bullet$ biochip $\bullet$ cytoskeleton $\bullet$ deformability $\bullet$ dendritic cells $\bullet$ dielectrophoresis $\bullet$ stretching

Cellular differentiation and maturation are commonly accompanied by changes in the actin cytoskeleton and these changes should be monitored accurately without disrupting the cycles of actin polymerization and depolymerization. Changes in the deformation behavior of cells are recognized as a valid biomarker for the detection of phenotypic differences. The link between biomechanics and human diseases has provided an improved understanding of organ and tissue function [1]. Several microtechnologies have been used to study mechanical deformations of biological cells and subcellular structures; for example, the influence of malaria-inducing Plasmodium falciparum on the stiffness and biomechanics of red blood cells has been studied [2]. Alterations in cell mechanics by reorganization of the actin cytoskeleton could be observed in correlation with strain-specific rubella virus phenotypes [3]. Deformability of cancer cells is relevant to establish an understanding of metastasis through dissociation from the primary tumor site [4]. A variety of experimental methods have been used to extract the mechanical properties of healthy cells and their diseased counterparts. Atomic force microscopy on single cells is a commonly used contact-dependent technique in which the extent of the measured deformation of the cantilever provides an indication of the diseased state of cancerous cells and parasite-infected cells [5-8]. Micropipette aspiration is another conventional technique to perform measurements for cell mechanics and reveal disease dynamics at the single cell level $[9,10]$. The limitation of using a single pipette - its low throughput - has been overcome with the use of microfluidics by means of parallel microchannels with a constriction such that cells are measurably deformed when passing through it [11]. A laser-enabled manipulation setup, commonly referred to as optical tweezers, can also be used in mechanical deformation studies of single cells; however, this approach cannot scale easily to accommodate large cell numbers [12,13]. Other techniques used to evaluate cell stiffness include the use of microfabricated microposts for measuring traction forces, pneumatic or piezoelectric deformation of flexible membranes with cells cultured on them and the use of microplates for compression followed by the characterization of stress-strain relationships [14-18]. Cell deformability can also be quantified in a microfluidic chip by exposing cells to hydrodynamic shear stress and pressure [19].

In this paper we report an electrical method for characterizing the biomechanical properties of dendritic cells (DCs) using dielectrophoretic forces. DCs are a vital part of the immune system and possess the ability to transform from immature antigen-capturing 


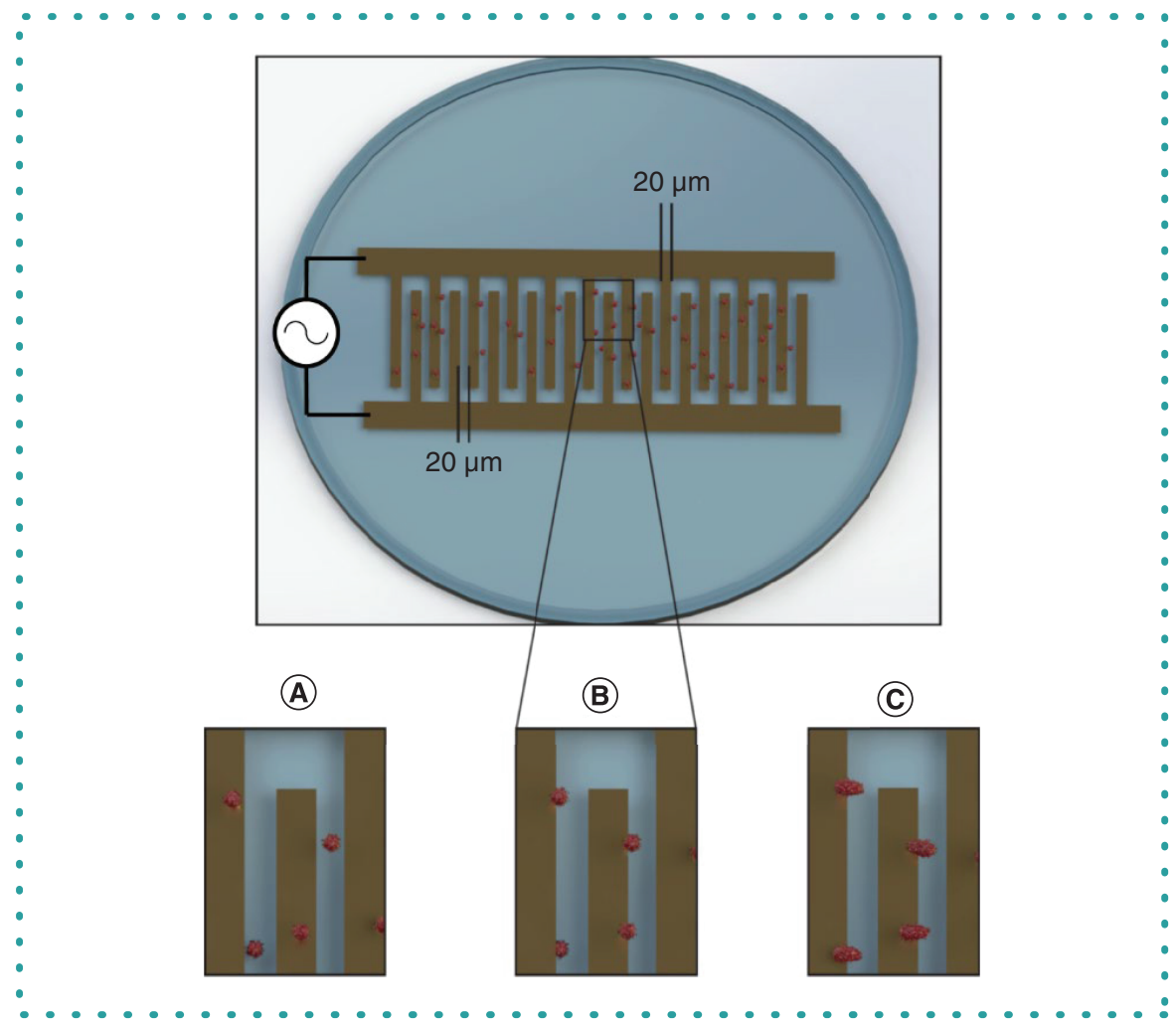

Figure 1. Schematic of the interdigitated electrode setup developed for dielectrophoretic biomechanical characterization. (A) The cells are randomly distributed over the electrodes prior to the application of an AC voltage. (B) The cells are immobilized on the electrode edge using positive dielectrophoresis. (C) The cells are stretched along the electric field lines upon increasing the electric voltage.

cells to mature antigen-presenting cells [20]. DCs are derived from the bone marrow and are usually present as immature cells within various bodily tissues, predominantly in the lymphoid organs [21]; they are also found in nonlymphoid tissues, particularly those that interface with the environment, such as skin, lung and gastrointestinal tract [22]. The maturation process is triggered by the internalization of foreign antigens. Subsequently, the mature DCs migrate from the foreign antigen detection site to the lymph nodes to activate T lymphocytes and trigger the adaptive immune response [23]. A reported feature of DC maturation is modification of the actin cytoskeleton. This progression involves changes in the activation status of signaling proteins and actin-controlling proteins, resulting in the downregulation of antigen capture and increased motility for migration to the T lymphocytes. Several studies have concluded that as a result of DC maturation, the DC cortex is modulated, resulting in T-cell activation. Blumenthal et al. utilized atomic force microscopy to physically measure the stiffness of immature and mature DCs derived from murine bone marrow [24]. The average Young's modulus of immature DCs was experimentally determined to be $2.2 \mathrm{kPa}$, whereas the mature DCs were significantly stiffer, with an average Young's modulus of $3.5 \mathrm{kPa}$. A recent report, using an artificial biosystem representing physical interaction between DCs and T cells, further pointed out a differential T lymphocyte response to varying simulated DC stiffnesses [25]. These measurements validated the hypothesis and contribute to the understanding that stiffness of the DC cortex is a trait sensed by $\mathrm{T}$ cell populations and assists in triggering T-cell activation.

Dielectrophoresis (DEP)-based stretching [26-30] is an electric field-based methodology which presents an alternative to conventional approaches such as micropipette aspiration and atomic force microscopy. This work utilizes nonuniform electric fields generated by interdigitated electrodes to stretch DCs in a direction parallel to the field lines, as shown in Figure 1. The primary advantage of this method is that it does not rely on physical contact to induce cellular elongations of several micrometers. Additionally, the large footprint of the interdigitated electrodes allows multiple cells to be elongated simultaneously and captured within the microscope's field of view. Measurement of filamentous actin (F-actin) was carried out using phalloidin conjugated to Alexa Fluor ${ }^{\circledR} 488$ and indicated a direct correlation between F-actin and deformability observed in DCs. 


\section{Materials \& methods}

\section{Cell culture, differentiation \& cytometry}

The human leukemia monocytic cell line (THP-1) was maintained in RPMI-1640 medium supplemented with 2 mM L-glutamine, $1 \%$ HEPES, $1 \%$ sodium pyruvate, $1 \%$ penicillin/streptomycin, $0.01 \% \beta$-mercaptoethanol and $10 \%$ heat-inactivated fetal bovine serum under standard cell culture conditions at $37^{\circ} \mathrm{C}, 5 \% \mathrm{CO}_{2}$ and $95 \%$ humidity. All reagents were obtained from Invitrogen, Karlsruhe, Germany.

DCs were differentiated from THP-1 cells as previously reported [31]. Briefly, $1 \times 10^{6}$ cells were cultivated in RPMI-1690 medium without fetal bovine serum, supplemented with $100 \mathrm{ng} / \mathrm{ml} \mathrm{GM-CSF}$ and $200 \mathrm{ng} / \mathrm{ml} \mathrm{IL-4}$ for immature DCs (iDCs), or $20 \mathrm{ng} / \mathrm{ml}$ TNF- $\alpha$ and $200 \mathrm{ng} / \mathrm{ml}$ ionomycin for mature DCs (mDCs). All cytokines and media supplements were supplied from Biolegend, USA. Cells were cultured in the differentiation media for 3 days under standard cell culture conditions at $37{ }^{\circ} \mathrm{C}, 5 \% \mathrm{CO}^{2}$ and $95 \%$ humidity.

To reduce the amount of F-actin in the cell, differentiated DCs were treated with $5 \mu \mathrm{M}$ of cytochalasin B (cyto-B; Tocris Bioscience; USA) for $2 \mathrm{~h}$. Cell morphology was imaged using EVOS FLoid Cell Imaging Station (Invitrogen, USA). Afterward, cells were detached using TrypLE ${ }^{T M}$ solution (Gibco, USA) and used for DEP experiment and F-actin quantification.

For quantitative analysis of F-actin, cyto-B-treated and untreated DCs were firstly stained with phalloidin conjugated with Alexa Fluor 488 (diluted 1:250 with cell culture media; Invitrogen) for 30 min under standard cell culture conditions. Afterward, cells were centrifuged and the cell pellet was resuspended in cell culture medium. The F-actin amount was analyzed by mean fluorescence signal intensity of phalloidin using flow cytometry (Attune, Invitrogen). The obtained results were quantified using FlowJo (BD, USA). The experiment was performed at least in triplicate.

\section{Dielectrophoretic medium}

Prior to performing DEP experiments, the cells were suspended in an iso-osmotic cell suspension medium for DEP cell stretching studies. The medium was prepared by adding $3 \mathrm{~g} / \mathrm{I}$ glucose and $85 \mathrm{~g} / \mathrm{I}$ sucrose to DI water. Conductivity was adjusted to $30 \mathrm{mS} / \mathrm{m}$ with phosphatebuffered saline, verified using a conductivity meter and the $\mathrm{pH}$ adjusted to 7.4 with $\mathrm{NaOH} / \mathrm{HCl}$. The osmolality of the final medium was $310 \mathrm{mOsm} / \mathrm{kg}$, verified with an osmometer.

\section{Dielectrophoretic device \& measurements}

The developed device to generate the DEP forces is a gold interdigitated microelectrodes array patterned on glass $(20 \mu \mathrm{m}$ width and $20 \mu \mathrm{m}$ spacing). It was fabricated using standard photolithography with $100 \mathrm{~nm}$ of gold and $5 \mathrm{~nm}$ of chrome, and occupies an approximate total area of $2 \mathrm{~cm}^{2}$. The electrodes were connected to an amplifier (Mini Circuits ZHL-5 W-1, 5-500 MHz), which in turn received its signal from a function generator (Keysight N5172B EXG X-Series). A sinusoidal voltage of 3.6 $\mathrm{V}_{\mathrm{pk} \text {-pk }}$ was applied to the electrodes to immobilize the suspended cells on the electrode edges. Once the cells were immobilized, stretching was performed by increasing the voltage to $20 \mathrm{~V}_{\mathrm{pk}}{ }^{-\mathrm{pk}}$. The $\mathrm{AC}$ frequency of the sinusoidal signal for immobilization and stretching was $10 \mathrm{MHz}$.

We recorded the dielectrophoretic cell stretching experiments using a Nikon LV100 upright microscope equipped with a camera. Reflected light microscopy was used to monitor DEP-induced cell stretching. In the case of reflection microscopy, the gap between the electrodes will appear darker; this was easily overcome by coating the underside of the glass substrate with either gold or a thin reflective sheet of aluminum, enabling light to reflect back into the objective and thereby illuminating every region of the cell.

The cell stretching measurements were carried out using ImageJ ( $\mathrm{NIH}, \mathrm{MD}$, USA). The examined cells were manually marked and numbered using the Image J software and then a digital ruler was used to measure the normal cell diameter and the elongated cell length post-stretching. The normal cell diameter was measured after immobilization of the suspended cells with the application of $3.6 \mathrm{~V}_{\mathrm{pk} \text {-pk }}$. This low voltage enables the cells to maintain sphericity without any apparent deformation. Within a few seconds of immobilization, the voltage was stepped up to $20 \mathrm{~V}_{\mathrm{pk} \text {-pk, }}$ resulting in rapid cell elongation within a few seconds to reach a stable fully stretched state. Image $J$ was then used to measure the major axis of the deformed cells that resembled an ellipse. The cell elongation length ( $\Delta \mathrm{I})$ was obtained by subtracting the major axis from the original cell diameter

The graphs and data were imported into MATLAB and plotted. The finite element simulations required to understand the process were performed using COMSOL Multiphysics. The boundary conditions were zero charge everywhere except on the electrodes, where a voltage was applied. The adjacent electrodes were given, in the simulations, a static potential difference of $10 \mathrm{~V}$ (peak).

\section{Results \& discussion}

After loading the cell suspension onto the electrodes, the cells moved randomly through the liquid by means of convection. When cells sediment near the microelectrode substrate, the dielectrophoretic force attracts them toward the nearest electrode edge, where they are immobilized. The time averaged DEP force on a cell in three dimensions can be given by [32]:

$$
F_{D E P}=\pi \varepsilon_{m} \operatorname{Re}[C M] R^{3} \nabla E^{2}
$$

where $R$ is the radius of the cell, $\varepsilon_{m}$ is the absolute permittivity of the medium, $\nabla E^{2}$ is the gradient of the square of the electric field acting on a cell in three dimensions and CM is the Clausius-Mossotti factor. If the real part of CM is positive, particles move toward the regions of high electric field experiencing what is termed positive DEP, which is employed in these experiments. 


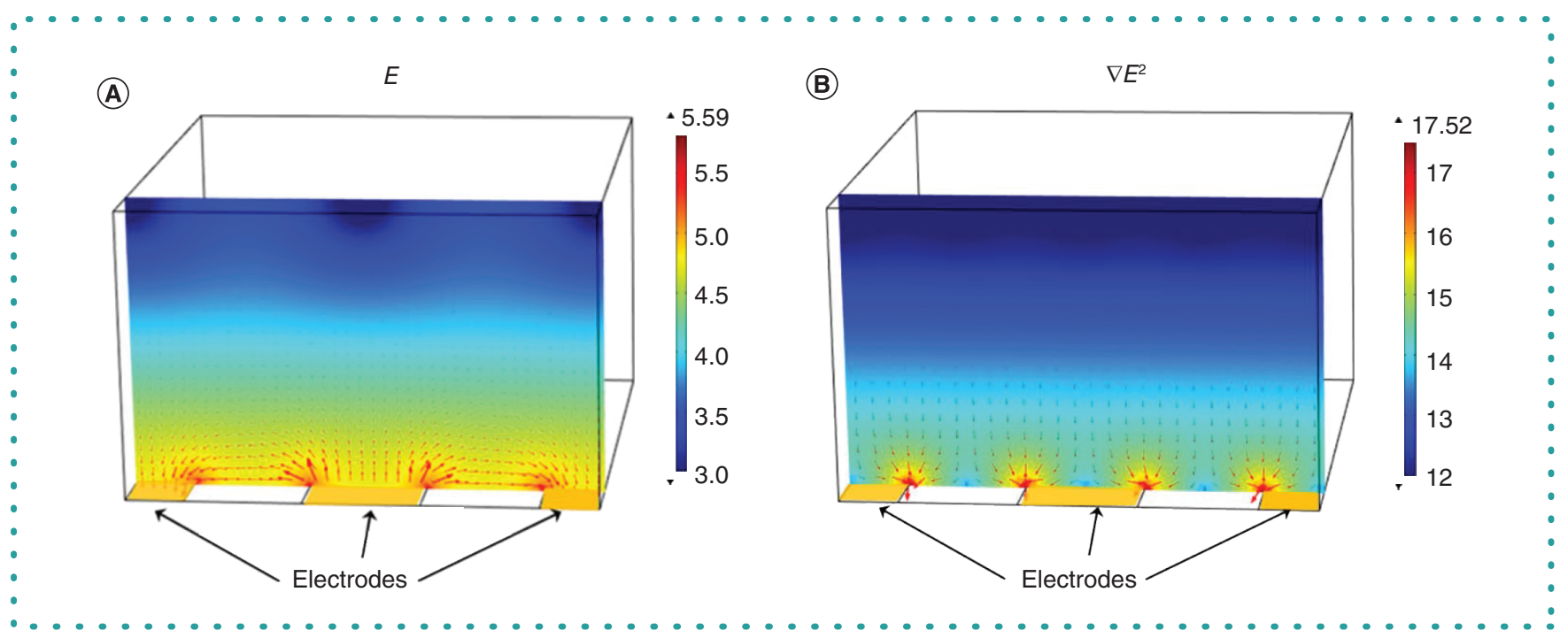

Figure 2. Finite element simulation of the microelectrode setup for a voltage of $3.6 \mathrm{~V}_{\mathrm{pk}-\mathrm{pk}}$. The electrodes are depicted by the gold structures at the bottom of the schematics. (A) Color plot shows the Electric Field magnitude $\left(\mathrm{V} / \mathrm{m}, \log _{10}\right.$ scale). The arrows indicate the direction of the field lines. (B) Color plot shows the $\nabla E^{2}$ magnitude $\left(\mathrm{V}^{2} / \mathrm{m}^{3}, \log _{10}\right.$ scale). The arrows represent the direction of the positive dielectrophoretic forces toward the electrode edges.

The electric field between the electrodes induces cell stretching. The finite element simulations of the electric field acting on the cell are shown in Figure 2A. The field arrows represent a stationary field, whereas the actual AC applied field will alternate. The magnitude is represented by the color plot with a maximum $\log _{10}$ value of $5.59 \mathrm{~V} / \mathrm{m}$ around the edges of the electrode; it decays to a $\log _{10}$ value of $3 \mathrm{~V} / \mathrm{m}$ at a height of $50 \mu \mathrm{m}$.

The arrows in Figure 2B show the direction of the dielectrophoretic force toward the microelectrodes along the gradient of the electric field. The color plot indicates the magnitude of $\nabla E^{2}$ with a maximum $\log _{10}$ value of $17.5 \mathrm{~V}^{2} / \mathrm{m}^{3}$ around the edges of the electrode; it decays to a $\log _{10}$ value of $12 \mathrm{~V}^{2} / \mathrm{m}^{3}$ at a height of $50 \mu \mathrm{m}$.

Net force calculations using the dipole approximation are valid in this electrode configuration due to the spatial dimensions of the electrode system and the cells. The geometric influence of the DEP force is given by $\nabla E^{2}$ as shown in the COMSOL simulation plots. The complex Maxwell stress tensor is a more rigorous approach in which the stress tensor is integrated over the cell surface to provide stress values on the cell suspension boundary, thereby indicating the pulling and pushing of the membrane resulting in trapping or the observed elongation. However, in most cases, the force derived from Maxwell stress tensor calculations is consistent with those obtained from the dipole approximation. The instantaneous DEP force can be obtained using the dipole approximation given by equation 1. For iDCs and mDCs, the average cell radius $(R)$ is measured to be approximately $6.75 \mu \mathrm{m}$. The permittivity of the aqueous medium, $\varepsilon_{m}$, can be assumed to be $79 \times 8.85 \times 10^{-12}$. During DEP, the Clausius-Mossotti function Re [CM] has values mathematically constrained between -0.5 and 1.0. For positive DEP of viable spherical cells with intact membranes suspended in a medium with ionic conductivity of $30 \mathrm{mS} / \mathrm{m}$, the $R e[C M]$ can be assumed to be about 0.65 [33]. In the experiments reported here, the center of the trapped cell is positioned at a distance of one cell radius from the electrode edge. At a distance of $6.75 \mu \mathrm{m}$ from the electrode edge, the term $\nabla E^{2}$ has been determined using COMSOL to be roughly $5 \times 10^{14}$ and $1.5 \times 10^{16}$ for a voltage of $3.6 \mathrm{~V}_{\mathrm{pk} \text {-pk }}$ and $20 \mathrm{~V}_{\mathrm{pk}-\mathrm{pk}}$, respectively. The corresponding net forces for a voltage of $3.6 \mathrm{~V}_{\mathrm{pk}-\mathrm{pk}}$ and $20 \mathrm{~V}_{\mathrm{pk}-\mathrm{pk}}$ are $0.21 \mathrm{nN}$ and $6.5 \mathrm{nN}$ respectively; these values are in the same range as that previously reported [34]. This net force can be regarded as a resolved point force at the geometric center of the cells and is largely accurate at the instant of application prior to cell deformation while the cells maintain their sphericity. However, this resultant central force can be decomposed and distributed nonuniformly over the cell membrane surface in three dimensions that can result in cellular elongation.

The experiment was initiated by loading $20 \mu \mathrm{l}$ of cell suspension on the electrode substrate and then covering it with a coverslip which rested on a 100- $\mu \mathrm{m}$-high spacer to limit convective flow. An AC voltage of $3.6 \mathrm{~V}_{\mathrm{pk}-\mathrm{pk}}, 10 \mathrm{MHz}$, was then applied, which did not result in any quantifiable deformation, but attracted the cells toward the electrode edge and immobilized them by means of positive DEP (Figure $3 \mathrm{~A}$ \& B). Subsequently, the voltage was switched to $20 \mathrm{~V}_{\mathrm{pk}-\mathrm{pk}}, 10 \mathrm{MHz}$, to stretch the cells and maximum elongation was observed in under $10 \mathrm{~s}$ of electric field application. Prior to electrical experimentation, the iDCs and mDCs were suspended in a pH and osmolarity adjusted buffer, but despite this, the exposure of cells to a low ionic conductivity solution was limited to below $3 \mathrm{~h}$ to maintain cell viability and function. The two representative cells from the iDC and $\mathrm{MDC}$ population exhibited a significant difference in electrical deformability.

These experiments were conducted in an aqueous ionic fluid, and hence it is vital to consider the influence of other electrokinetic forces that might contribute to shear flow-induced stretching. In addition to DEP, the other most frequently reported phenomena are AC 


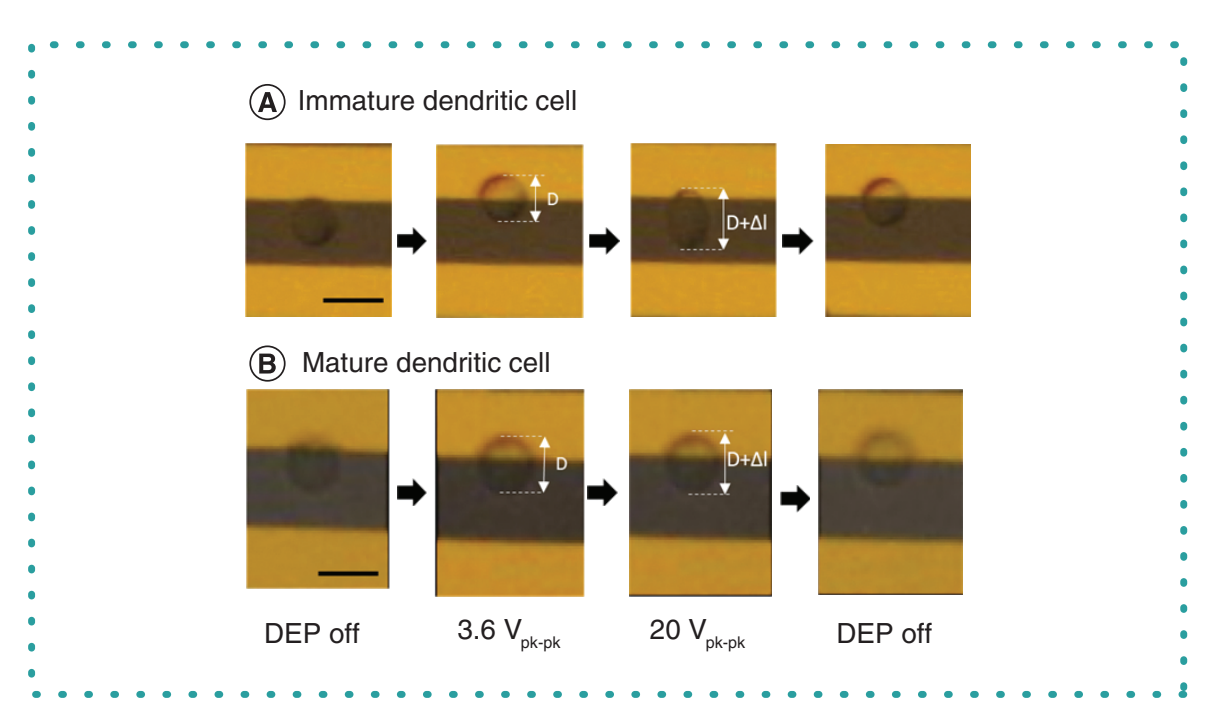

Figure 3. Dielectrophoretic stretching performed on (A) an immature dendritic cell and (B) a mature dendritic cell, subjected to strong electric fields from the interdigitated electrode chip. The four steps (left to right) are the DEP OFF state prior to cell stretching, cell immobilization at the electrode edge with a voltage of $3.6 \mathrm{~V}_{\mathrm{pk}-\mathrm{pk}} / 10 \mathrm{MHz}$, cell stretching with a voltage of $20 \mathrm{~V}_{\mathrm{pk}-\mathrm{pk}} / 10 \mathrm{MHz}$, and the DEP OFF state post-cell stretching. The cells depicted here are detached from their culture substrate and loaded to the device as a cell suspension for deformation measurements. Prior to stretching, cells adopt a spherical form.

electro-osmotic flow and electrothermal flow [35]. In the case of AC electro-osmosis, a force on the ions in the electrical double layer leads to fluid movement observed around the electrode edges. The velocity of fluid movement as a result of AC electro-osmosis is strongly dependent on the AC frequency and ionic medium conductivity [36]. For a medium conductivity of $30 \mathrm{mS} / \mathrm{m}$ and low AC frequencies of $\approx 10^{4} \mathrm{~Hz}$, ions can respond rapidly to the electric field, resulting in significant fluid flow velocities. However, for a medium conductivity of $30 \mathrm{mS} / \mathrm{m}$ and the high frequency of $10^{7} \mathrm{~Hz}$ employed in our experiments, AC electro-osmotic velocity tends to be zero because there is not enough time for the ionic charges to follow the electric field; hence the effect of AC electro-osmosis is negligible. Electrothermal flow is a result of joule heating, which gives rise to conductivity and permittivity gradients within the fluid medium, resulting in an electrical body force driving fluid flow. Electrothermal flow is also strongly dependent on AC frequency and ionic medium conductivity [37], reaching a maximum at high frequency and high conductivity. With the low medium conductivity of $30 \mathrm{mS} / \mathrm{m}$ used in our experiments, it is unlikely that electrothermal flow is a contributing factor.

For viable cells suspended in a medium of $30 \mathrm{mS} / \mathrm{m}$ conductivity, the applied frequency of $10 \mathrm{MHz}$ is optimized for both maximum positive DEP forces and subsequent electrodeformation. A lower AC electric field frequency $(0.1-1 \mathrm{MHz})$ is suboptimal because it can cause electrical damage to cells. This is as a result of the enlarged membrane field due to the potential drop induced across the extremely thin cell membrane. The membrane field $\left(E_{m}\right)$ can be written as a function of the field frequency ( $\left.\omega\right)$ [32]:

$$
E_{m}=\frac{1.5(R / d)|E| \cos \theta}{1+i \omega \tau}
$$

The factor $R / d$ is the ratio of the cell radius $R$ to the membrane thickness $d, \theta$ is the polar angle with respect to the field direction and $\tau$ is the time constant:

$$
\tau=\frac{R C_{m}}{\frac{2 \sigma_{i} \sigma_{e}}{\sigma_{i}+2 \sigma_{e}}+\frac{R \sigma_{m}}{d}}
$$

where $\sigma_{i}, \sigma_{m}$ and $\sigma_{e}$ are the conductivities of the cell interior (cytoplasm), cell membrane and extracellular medium, respectively. $C_{m}$ is the membrane capacitance. The magnitude of the amplified membrane field $\left(\left|E_{m}\right| /|E|\right)$ can be written as a function of the field frequency [38]. The membrane thickness $d$ can be approximated as $5 \mathrm{~nm}$, and the membrane field is generated parallel to the applied field (i.e., $\theta=0$ ). The value for $C_{m}$ for a viable cell was assumed to be approximately $14 \mathrm{mF} / \mathrm{m}^{2}[32,38]$. An experimental value of $30 \mathrm{mS} / \mathrm{m}$ was used for the conductivity of the cell suspending medium, and values of $0.7 \mathrm{~S} / \mathrm{m}$ and $5 \times 10^{-7} \mathrm{~S} / \mathrm{m}$ were assumed for the conductivities of the cell interior and membrane [32,38]. The value of the external field was $3.1 \times 10^{5} \mathrm{~V} / \mathrm{m}$ for an applied voltage of $20 \mathrm{~V}_{\mathrm{pk} \text {-pk }}$. Using these values, a time constant $(\tau)$ value of $1.69 \times 10^{-6} \mathrm{~s}$ was obtained. This clearly demonstrates how the membrane field varies as a function of frequency. We can clearly observe from Figure 4 that the amplification of the external field in the membrane drops with increasing frequency, significantly minimizing cell membrane damage at a frequency of $10 \mathrm{MHz}$. Hence a frequency of $10 \mathrm{MHz}$ serves to induce cell elongation and minimize electric field-related cell destruction due to a weaker membrane field. 


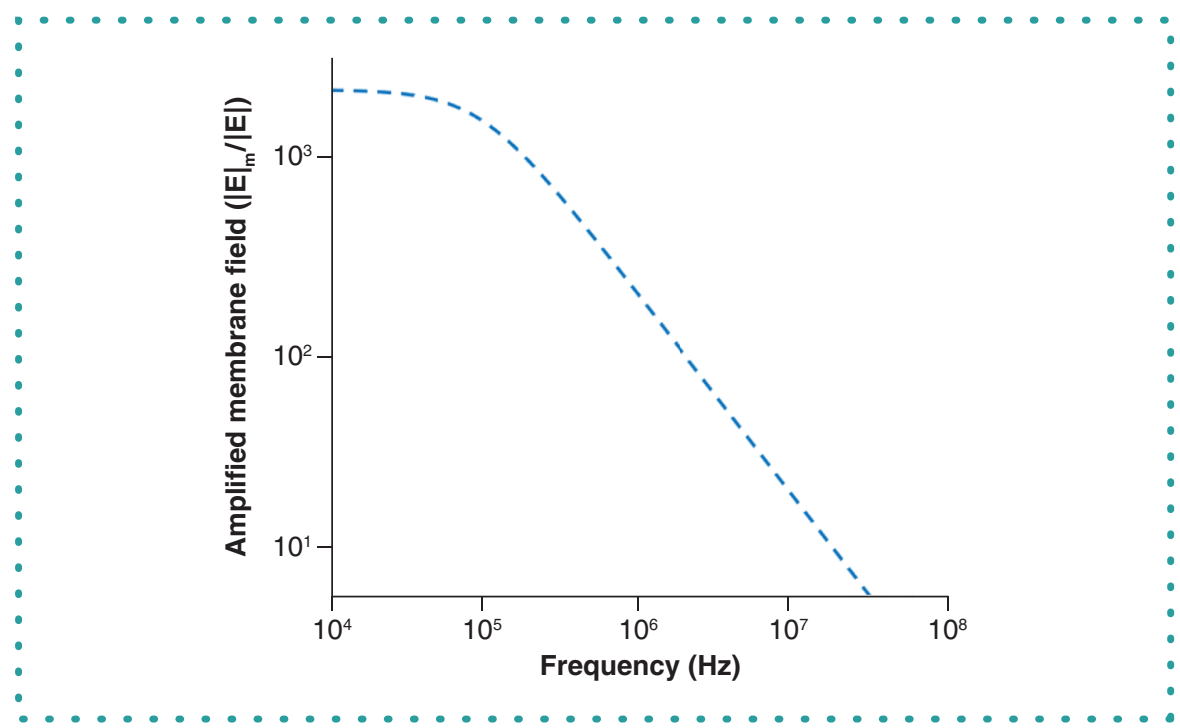

Figure 4. Amplified cell membrane field as a function of the applied AC frequency. Radius of the viable cell is $6.75 \mu \mathrm{m}$, the applied external field is $3.1 \times 10^{5} \mathrm{~V} / \mathrm{m}$, and the suspending medium conductivity is $30 \mathrm{mS} / \mathrm{m}$.

The deformation was quantified using the Elongation Ratio ( $E R=\Delta l /$ Cell Diameter), in other words the ratio between the cell elongation length $(\Delta l)$ along the field direction and the initial cell diameter. Approximately 30 cells for each condition were analyzed, as shown in Figure 5A. The average values and standard error obtained from 30 cells for each maturation state were analyzed. Untreated iDCs and mDCs had an ER of $0.192 \pm 0.029$ and $0.053 \pm 0.009$, respectively. This clearly demonstrates that iDCs under normal conditions are significantly less stiff than mDCs, and these measurements validate earlier findings using atomic force microscopy on the implications of cell stiffness in DCs [24].

To determine whether the distribution and architecture of actin was primarily responsible for deformability, we treated immature and mature DCs with the actin inhibition agent cyto-B, which limits actin polymerization and the interaction of actin filaments (Figure $5 \mathrm{~B}$ ). Cyto-B-treated iDCs and mDCs had ERs of $0.207 \pm 0.023$ and $0.193 \pm 0.027$, respectively, clearly indicating that treatment with cyto-B results in significant increase in the deformability of $\mathrm{mDCs}$ to approximately that of iDCs, whereas iDCs showed insignificant changes in deformability characteristics after treatment.

To confirm the result from the DEP, we demonstrated that cyto-B affects F-actin in mDCs while in culture. Figure $5 \mathrm{C}$ shows representative images of cells both with and without cyto-B treatment. It was observed that mDCs exhibited pronounced dendrites when compared with the iDCs. After treatment with cyto-B, both cells demonstrated a rounded morphology, suggesting a reduction of actin. To confirm the visual observation, we performed flow cytometry analysis of F-actin by staining with phalloidin conjugated with Alexa Fluor 488. As shown in Figure 5D, we found that iDCs (664 $\pm 110 \mathrm{au})$ exhibited significantly lower F-actin when compared with mDCs $(3760 \pm 326 \mathrm{au})$. After treatment with cyto-B, the amount of F-actin was reduced in both models, but the effect in mDCs $(2287 \pm 153$ au) was much stronger than in iDCs (490 $\pm 47 \mathrm{au})$. By comparing this data with the DEP measurement, it could be shown that lowering F-actin amount results in higher ER values. The F-actin amount and elongation measurement were well correlated. Our result suggests the role of F-actin in maintaining cellular mechanics and matches with results from cell deformation cytometry studies [3].

In this study we describe a rapid experimental setup to determine the biomechanical properties of human cell line-based DCs before and after maturation. The developed noncontact DEP technique, utilizing a nonuniform electric field, was employed to stretch DCs along the electric field lines. This offered considerable forces for sufficient elongation of multiple cells simultaneously. Quantification of filamentous actin was carried out to provide direct correlation between F-actin expression and deformability observed in mDCs. The two states of DCs were also treated with cyto-B to inhibit actin activity, and the findings clearly indicate that this drug treatment results in a significant increase in the deformability of $\mathrm{mDCs}$, to a range similar to that observed in iDCs. This clearly establishes the role that actin plays in the maturation process of DCs and their resulting migration and adaptive immune response. We also demonstrated that DEP has the sensitivity to distinguish phenotypic differences in cells. The developed system is versatile, simple and rapid, and can be applied to other cell types and models for studying the effect of relevant drug treatments or diagnostic markers.

\section{Future perspective}

Cell stretching using DEP is a valuable method for stiffness characterization based on the structural properties of the cellular membrane and cytoskeleton. Electromechanical deformation can be performed to track the physiological changes occurring in cells as a result of diseases. One longer-term application that can be envisioned is the implementation of a deformability assay to study cells within 
(A)

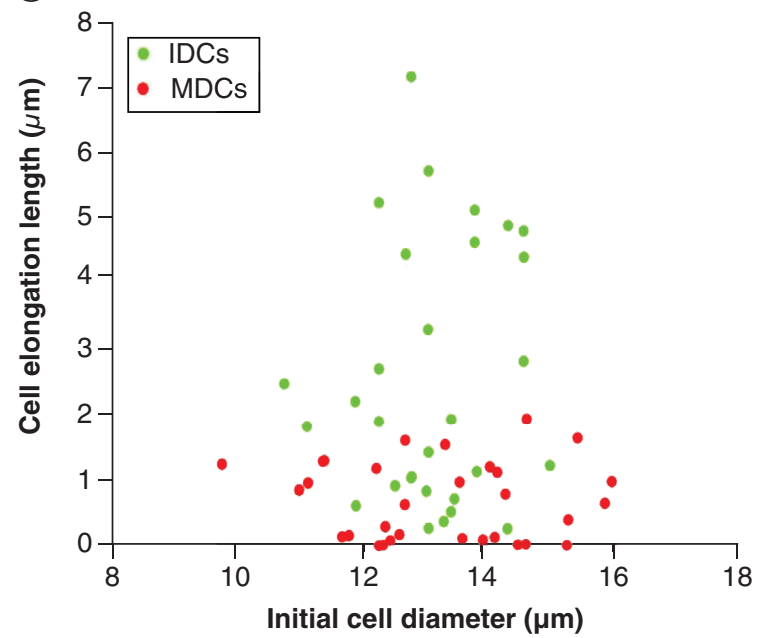

(C)
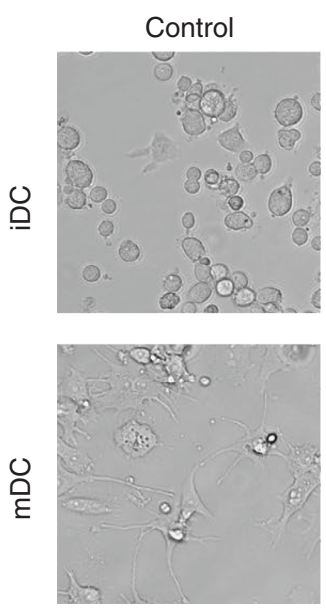

(B)

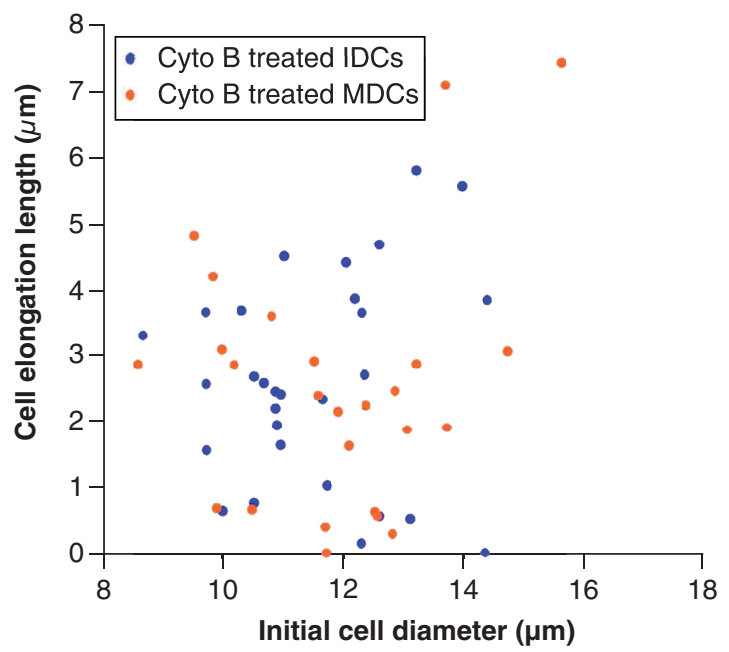

(D) Quantification of f-actin amount

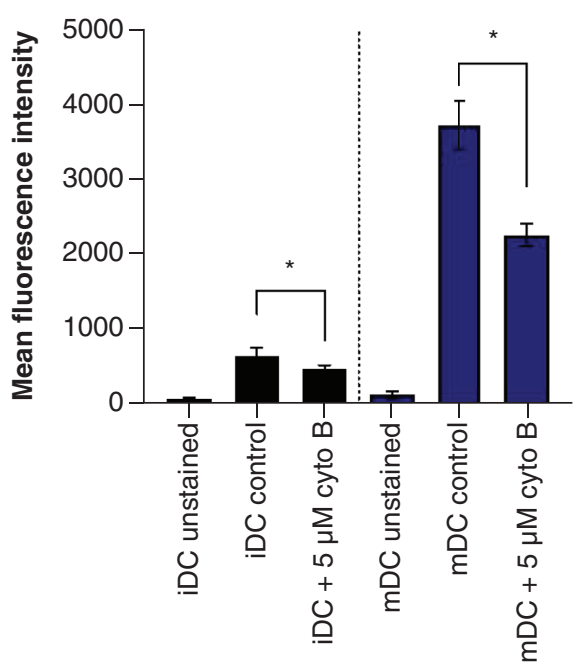

Figure 5. Deformability and actin quantification pre- and post-treatment of immature and mature dendritic cells with cytochalasin B. (A) Scatter plot describing the stretching characteristics of untreated iDCs and mDCs. (B) Scatter plot describing the stretching of cytochalasin B-treated iDCs and mDCs. (C) Representative image of iDCs and mDCs in an adherent state with and without cytochalasin B treatment. (D) Flow cytometery analysis of F-actin by staining using phalloidin conjugated with Alexa Fluor 488. Mean fluorescence intensity is presented as mean \pm standard devation. *p $<0.05$.

CytoB; Cytochalasin B; iDC: Immature dendritic cell; mDC: Mature dendritic cell.

biological fluids such as blood and urine. In addition, cells obtained can also be analyzed for diseases such as cancer and chronic intestinal diseases.

\section{Author contributions}

This project was conceived and designed by A Menachery and M Qasaimeh. The experiments and data analysis were conducted by $\mathrm{A}$ Menachery, J Sapudom, A Vembadi and A Alatoom. The manuscript was written by A Menachery, J Sapudom and A Vembadi, and edited by J Teo and M Qasaimeh. 


\section{Acknowledgments}

The authors acknowledge the technical support from the Core Technology Platforms at NYU Abu Dhabi and thank C Johnson for designing the schematic in Figure 1.

\section{Financial \& competing interests disclosure}

This work was supported by Seed Grant (AJF2018085) from Al Jalila Foundation, Dubai, UAE, the New York University Abu Dhabi Grants for Publication Program, and the Research Enhanced Fund (RE266) from New York University Abu Dhabi. The authors have no other relevant affiliations or financial involvement with any organization or entity with a financial interest in or financial conflict with the subject matter or materials discussed in the manuscript apart from those disclosed.

No writing assistance was utilized in the production of this manuscript.

\section{Open access}

This work is licensed under the Attribution-NonCommercial-NoDerivatives 4.0 Unported License. To view a copy of this license, visit http://creativecommons.org/licenses/by-nc-nd/4.0/

\section{References}

1. Cox TR, Erler JT. Remodeling and homeostasis of the extracellular matrix: implications for fibrotic diseases and cancer. Dis. Model. Mech. 4(2), 165-178 (2011).

2. Dépond $M$, Henry B, Buffet $P$, Ndour A. Methods to investigate the deformability of RBC during malaria. Front. Physiol. 10, 1613 (2020).

3. Kraeter M, Sapudom J, Bilz N, Pompe T, Guck J, Claus C. Alterations in cell mechanics by actin cytoskeletal changes correlate with strain-specific rubella virus phenotypes for cell migration and induction of apoptosis. Cells 7(9), 136 (2018)

4. Deliorman $\mathrm{M}$, Janahi FK, Sukumar $\mathrm{P}$ et al. A microfluidic-atomic force microscopy platformfor the capture and nanomechanical characterization of circulating tumor cellsin prostate cancer. Microsystem. Nanoeng. 6(20), DOI:10.1038/s41378-020-0131-9 (2020)

5. Deng X, Xiong F, Li X et al. Application of atomic force microscopy in cancer research. J. Nanobiotechnol. 16(1), 102 (2018).

6. Rebelo L, de Sousa J, Filho J, Radmacher M. Comparison of the viscoelastic properties of cells from different kidney cancer phenotypes measured with atomic force microscopy. Nanotechnology 24(5), 055102 (2013)

7. Shi H, Li A, Yin J, Tan KSW. AFM study of the cytoskeletal structures of malaria infected erythrocytes. In: 13th International Conference on Biomedical Engineering. IFMBE Proceedings, vol 23. Goh JCH, Lim CT (Eds). Springer, Berlin/Heidelberg, Germany (2009).

8. Shi H, Liu Z, Li A et al. Life cycle-dependent cytoskeletal modifications in Plasmodium falciparum infected erythrocytes. PloS ONE 8(4), e61170-e61170 (2013).

9. Di Cerbo A, Rubino V, Morelli F et al. Mechanical phenotyping of K562 cells by the Micropipette Aspiration Technique allows identifying mechanical changes induced by drugs. Sci. Rep. 8(1), 1219 (2018)

10. Lee LM, Liu AP. The application of micropipette aspiration in molecular mechanics of single cells. J. Nanotechnol. Eng. Med. 5(4), 0408011-0408016 (2014).

11. Myrand-Lapierre M-E, Deng X, Ang R, Matthews K, Santoso A, Ma H. Multiplexed fluidic plunger mechanism for the measurement of red blood cell deformability. Lab Chip 15(1), 159-167 (2014).

12. Guo HL, Liu CX, Duan JF et al. Mechanical properties of breast cancer cell membrane studied with optical tweezers. Chin. Phys. Lett. 21, 2543-2546 (2004).

13. Lim CT, Dao M, Suresh S, Sow CH, Chew KT. Large deformation of living cells using laser traps. Acta Mater. 52, 1837-1845 (2004).

14. Desprat N, Guiroy A, Asnacios A. Microplates-based rheometer for a single living cell. Rev. Sci. Instrum. 77,055111 (2006).

15. Kamble H, Barton M, Jun M, Park S, Nguyen N-T. Cell stretching devices as research tools: engineering and biological considerations. Lab Chip 16(17), 3193-3203 (2016).

16. Kreutzer J, Viehrig M, Pölönen R-P et al. Pneumatic unidirectional cell stretching device for mechanobiological studies of cardiomyocytes. Biomech. Model. Mechanobiol. 19(1), 291-303 (2019).

17. Lam RHW, Weng S, Lu W, Fu J. Live-cell subcellular measurement of cell stiffness using a microengineered stretchable micropost array membrane. Integr. Biol. (Camb.) 4(10), 1289-1298 (2012).

18. Ribeiro AJS, Denisin AK, Wilson RE, Pruitt BL. For whom the cells pull: hydrogel and micropost devices for measuring traction forces. Methods $94,51-64$ (2016).

19. Otto O, Rosendahl P, Mietke A et al. Real-time deformability cytometry: on-the-fly cell mechanical phenotyping. Nat. Methods 12(3), (2015).

20. Stockwin LH, McGonagle D, Martin IG, Blair GE. Dendritic cells: immunological sentinels with a central role in health and disease. Immunol. Cell Biol. 78(2), 91-102 (2000).

21. Alvarez D, Vollmann EH, von Andrian UH. Mechanisms and consequences of dendritic cell migration. Immunity 29(3), 325-342 (2008).

22. Witzel I-I, Nasser R, Garcia-Sabaté A et al. Deconstructing immune microenvironments of lymphoid tissues for reverse engineering. Adv. Healthc. Mater. 8(4), e1801126 (2018).

23. Worbs T, Hammerschmidt S, Förster R. Dendritic cell migration in health and disease. Nat. Rev. Immunol. 17(1), 30-48 (2016).

24. Blumenthal D, Chandra V, Burkhardt J. Mouse T cell priming is enhanced by maturation-dependent stiffening of the dendritic cell cortex. Elife 27 , e55995 (2020).

25. Alatoom A, Sapudom J, Soni P, Mohamed W, Garcia-Sabaté A, Teo JCM. Artificial biosystem for modulation of interactions between antigen-presenting cells and T cells. Adv. Biosystems 4(7), e2000039 (2020).

26. Bai G, Li Y, Chu H et al. Characterization of biomechanical properties of cells through dielectrophoresis-based cell stretching and actin cytoskeleton modeling. Biomed. Eng. Online 16(1), 41 (2017).

27. Du E, Dao M, Suresh S. Quantitative biomechanics of healthy and diseased human red blood cells using dielectrophoresis in a microfluidic system. Extreme Mech. Lett. 1, 35-41 (2014).

28. Guido I, Jaeger MS, Duschl C. Dielectrophoretic stretching of cells allows for characterization of their mechanical properties. Eur. Biophys. J. 40(3), 281-288 (2011).

29. Qiang Y, Liu J, Yang F, Dieujuste D, Du S. Modeling erythrocyte electrodeformation in response to amplitude modulated electric waveforms. Sci. Rep. 8(1), 10224 (2018).

30. Zhu B, Cai Y, Wu Z, Niu F, Yang H. Dielectrophoretic microfluidic chip integrated with liquid metal electrode for red blood cell stretching manipulation. IEEE Access 7, 152224-152232 (2019).

31. Sapudom J, Alatoom A, Mohamed W et al. Dendritic cell immune potency on 2D and in 3D collagen matrices. Biomater. Sci. 8(18), 5106-5120 (2020).

32. Menachery A, Pethig R. Controlling cell destruction using dielectrophoresis. IEE Proc. Nanobiotechnol. 152, 145-149 (2005).

33. Pethig R, Menachery A, Pells S, Sousa P. Dielectrophoresis: a review of applications for stem cell research. J. Biomed. Biotechnol. 2010, 182581 (2010).

34. Bai G, Li Y, Chu HK et al. Characterization of biomechanical properties of cells through dielectrophoresis-based cell stretching and actin cytoskeleton modeling. Biomed. Eng. Online 16(1), 41-41 (2017).

35. Morgan H, Green N. AC Electrokinetics: Colloids and Nanoparticles. Research Studies Press, Hertfordshire, UK (2003).

36. Ramos A, Morgan H, Green N, Castellanos A. AC electrokinetics: a review of forces in microelectrode structures. J. Phys. D: Appl. Phys. 31(18), 2338-2353 (1998).

37. Salari A, Navi M, Lijnse T, Dalton C. AC electrothermal effect in microfluidics: a review. Micromachines 10, 762 (2019).

38. Kotnik T, Miklavcic D. Second-order model of membrane electric field induced by alternating external electric fields. IEEE Trans. Biomed. Eng. 47(8), 1074-1081 (2000). 